\title{
e-Phaïstos
}

e-Phaïstos

Revue d'histoire des techniques / Journal of the history

of technology

VIII-2 | 2020

Aviation : émergence d'un complexe technique, 1900-1930

\section{Modelées ou moulées ? Les premières briques crues du Caucase au Néolithique}

Shaped by Hand or Moulded? The First Sun-dried Mud Bricks in the Southern

Caucasus Neolithic

Emmanuel Baudouin

\section{CpenEdition}

Journals

Édition électronique

URL : https://journals.openedition.org/ephaistos/7912

DOI : 10.4000/ephaistos.7912

ISSN : 2552-0741

Éditeur

IHMC - Institut d'histoire moderne et contemporaine (UMR 8066)

Référence électronique

Emmanuel Baudouin, « Modelées ou moulées ? Les premières briques crues du Caucase au

Néolithique », e-Phaïstos [En ligne], VIII-2 | 2020, mis en ligne le 27 octobre 2020, consulté le 17 septembre 2021. URL : http://journals.openedition.org/ephaistos/7912 ; DOI : https://doi.org/ 10.4000/ephaistos.7912

Ce document a été généré automatiquement le 17 septembre 2021.

Tous droits réservés 


\section{Modelées ou moulées ? Les premières briques crues du Caucase au Néolithique}

Shaped by Hand or Moulded? The First Sun-dried Mud Bricks in the Southern

Caucasus Neolithic

Emmanuel Baudouin

1 La genèse de la technique de la brique est un sujet bien documenté en Mésopotamie. Les différentes étapes évolutives de la brique modelée à la brique moulée sont attestées entre la fin du $9^{e}$ et le milieu du $7^{\mathrm{e}}$ millénaire av. J.-C. (Aurenche 1981; Aurenche 1993; Sauvage 1998; Sauvage 2001; Sauvage 2011). Pour le Caucase, les recherches sur les techniques de construction sont relativement récentes (Ioseliani 2017; Baudouin et al. 2018; Baudouin 2019). Dans cette région, les premiers établissements sédentaires apparaissent plus tardivement qu'en Mésopotamie à la fin $d u 7^{e}$ millénaire. Dès le milieu du XXe siècle, les recherches se sont focalisées sur les modalités d'installation de ces premières communautés. Deux hypothèses majeures s'affrontent: une genèse locale et autonome (Vavilov 1932; Amirkhanov 1987); l'apport des communautés de Mésopotamie par l'échange de matériaux et d'objets (Kiguradze 1986; Kushnareva 1997) ou d'une « colonisation » (Abibullaev 1959 ; Narimanov 1987).

2 L'étude de l'évolution des techniques architecturales s'inscrit dans cette dynamique de recherche. C'est un élément clé pour la compréhension du développement culturel et technique dans la région. Ces recherches sont d'autant plus importantes que l'architecture est un objet de la culture matérielle à forte inertie technique, soumis à des règles de circulation et d'échange originales : l'idée et les connaissances circulent et non l'objet fini ou la matière comme cela peut-être le cas pour d'autres artefacts. Dès lors, l'architecture doit être comprise comme l'élément moteur d'un réseau d'échanges particuliers où les acteurs de la transmission ne peuvent être que les constructeurs eux-mêmes. 
3 À partir de ce constat, plusieurs questions peuvent être posées. Quelles sont les techniques de façonnage de la brique attestées au Néolithique dans le Caucase? La brique moulée est-elle utilisée dès cette période? Si oui, peut-on définir une évolution locale des techniques, ou s'agit-il au contraire d'un apport des communautés mésopotamiennes? Enfin, quels sont les moyens à disposition de l'archéologue pour restituer les étapes de la chaîne opératoire à partir des vestiges matériels? Au regard des découvertes archéologiques récentes, il est possible d'avancer sur la question de l'évolution de la technique de la brique dans le sud du Caucase au Néolithique.

\section{Les attestations de la brique crue dans le Caucase au Néolithique}

4 La technique de la brique crue semble maitrisée dès l'installation des premières communautés sédentaires (fin $7^{\mathrm{e}}$ - début $6^{\mathrm{e}}$ millénaire) dans la moyenne vallée de la Kura, en Azerbaïdjan, et dans la plaine de l'Ararat, en Arménie (fig.1). Elle apparaît plus tardivement dans la plaine de Mil (autour de 5600 av. J.-C.), en même temps que les premières installations sédentaires. Sur plusieurs sites, la brique est utilisée en concomitance de la bauge ${ }^{1}$ (Baudouin 2019). Cette dernière technique, bien que minoritaire, continue d'être employée tout au long du Néolithique dans le Caucase alors qu'en Mésopotamie, on constate une diminution de son utilisation entre la fin du $7^{\mathrm{e}}$ et le milieu du $6^{\mathrm{e}}$ millénaire au profit de la brique crue (Baudouin en préparation). La composition de la terre à bâtir, que ce soit pour la brique ou la bauge, varie peu dans l'ensemble. Il s'agit d'un mélange de terre et d'eau auquel est ajouté un dégraissant souvent végétal - utile pour augmenter la cohésion de la terre - ou occasionnellement minéral. 
Fig.1 : Carte générale du Caucase avec les sites néolithiques mentionnés dans le texte et les différentes attestations de la brique

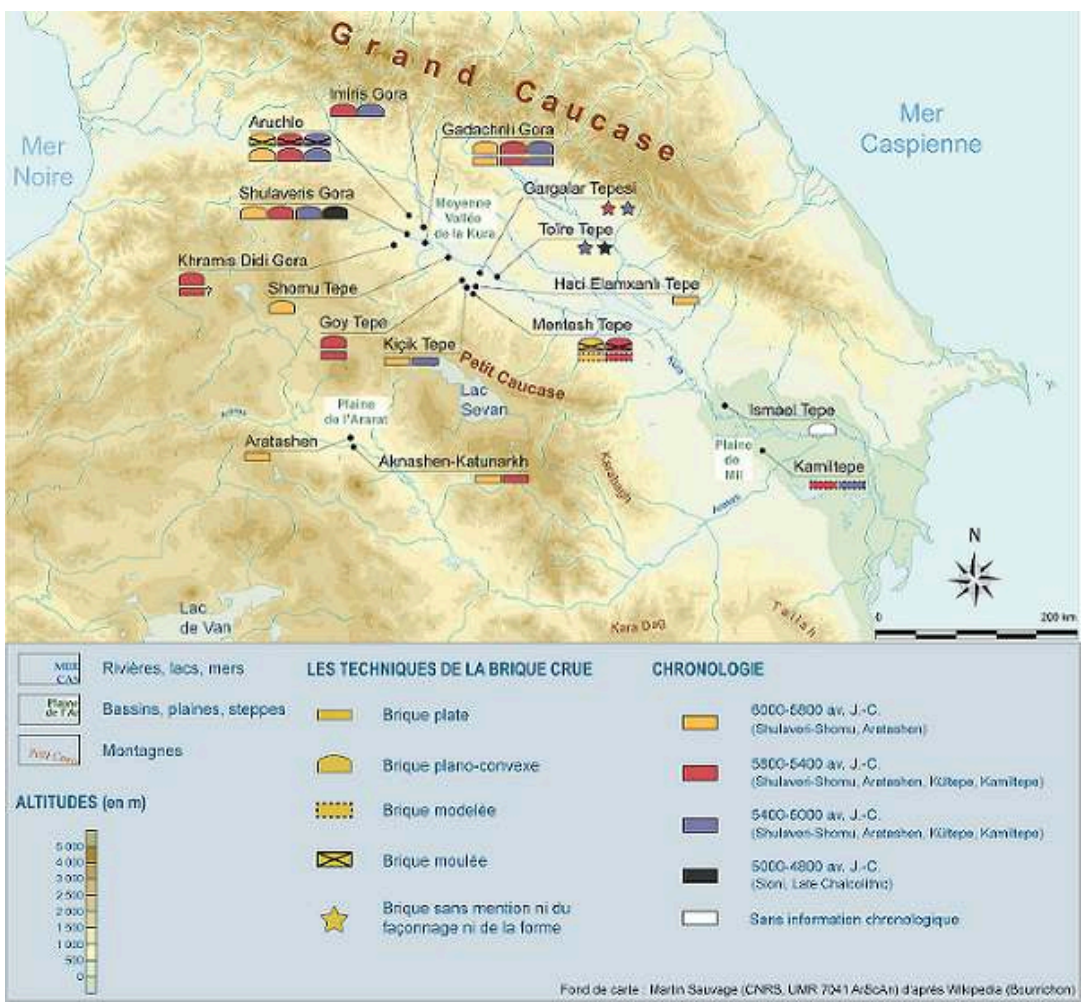

Dans la littérature archéologique, les descriptions font souvent état de la morphologie des briques (plate ou plano-convexe) mais rarement de la technique de façonnage (modelée et moulée principalement). Cependant, les nouvelles données archéologiques issues de la fouille des sites d'Aruchlo et de Mentesh Tepe ont confirmé l'usage du moule à brique dès le début du $6^{\mathrm{e}}$ millénaire.

Emmanuel Baudouin

Dans un premier temps, les critères morphologiques ont permis de distinguer deux ensembles régionaux avec, dans la vallée de l'Araxe, la production de briques plates et, dans la moyenne vallée de la Kura, celle de briques plano-convexes (Chataigner 1995; Kushnareva 1997). Une reprise récente des données archéologiques a démontré que l'aire de production des briques plates pouvait s'étendre à la moyenne vallée de la Kura et à la plaine de Mil où les attestations sont nombreuses durant tout le Néolithique (Baudouin 2019). En revanche, la zone de production des briques plano-convexes semble bien circonscrite à la moyenne vallée de la Kura, avec toutefois des attestations plus à l'est, dans la plaine du Karabagh (Ismail Tepe), mais toujours à proximité d'affluents de la Kura. De fait, la brique plano-convexe représente un important marqueur technoculturel (Chataigner 1995 :58).

Indépendamment de leur forme, les briques peuvent être classées selon trois catégories en fonction de leur dimension (Baudouin 2019:114, fig.5). Jusqu'au milieu du $6^{\mathrm{e}}$ millénaire, leur taille est variable, mais après cette période les modules tendent à se standardiser (Baudouin 2019 :113).

7 Si la forme des briques et leurs dimensions sont presque toujours mentionnées dans la littérature archéologique, ce n'est pas le cas du mode de façonnage. Seule Christine Chataigner (1995: 57-58) fait état de «briques modelées au profil en dos d'âne " pour désigner les briques plano-convexes de la Kura en référence aux briques proche-orientales produites au PPNA ( $9^{\mathrm{e}}-8^{\mathrm{e}}$ millénaires). 


\section{Chaîne opératoire et critères d'identification}

8 Selon la terminologie établie par Olivier Aurenche (2003:279, tab.1), deux procédés principaux de façonnage de la brique peuvent être distingués: le modelage et le moulage. Grâce aux recherches ethnoarchéologiques (Wulff 1966; Guest-Papamanoli 1978; Daune-Le Brun 2003; Darles et al. 2011 :20) et archéologiques (Sauvage 1998; Sauvage 2009) sur la brique et sa mise en œuvre, cette typologie peut être affinée et élargie à quatre procédés principaux: le modelage, le coupage, le pressage et le moulage (Tab.1). Pour ce dernier, au moins trois modes opératoires sont distingués en Orient : la technique dite «à coup d'eau » (utilisant un moule sans fond préalablement humidifié pour faciliter le démoulage), celle du «moule enfoncé » (ou à l'emportepièce) et celle "à coup de sable " (utilisant un moule à fond, amovible ou non, humidifié puis saupoudré de sable pour faciliter le démoulage). Comme résumé dans le tableau 1, la chaîne opératoire de ces différentes techniques varie en fonction des étapes de la confection.

D'après la littérature sur la question, il ressort que les critères d'identification d'une brique moulée sur le terrain archéologique peuvent être classés selon deux types: les critères primaires et les critères secondaires. Les premiers correspondent à l'observation de stigmates sur le matériau lui-même. Ils peuvent être distingués des critères secondaires, qui ne font pas référence à des étapes particulières de la chaîne opératoire, mais plutôt à un ensemble de paramètres empiriques (ci-dessous).

Parmi les critères primaires, deux étapes de la chaîne opératoire sont observables sur les briques si un démontage minutieux du mur est effectué lors de la fouille (Aurenche 1981 :64; Delougaz 1933:8; Tunca $1984: 122-123$ ). Premièrement, le raclage de l'excédent de terre disposé dans le moule crée des traces ${ }^{2}$ circulaires ou longitudinales sur la face supérieure de la brique (Fig.2 a-b). 
Fig.2 a-b : Mentesh Tepe, Azerbaïdjan (Chalcolithique, 5e millénaire). Traces de raclage sur une brique crue moulée

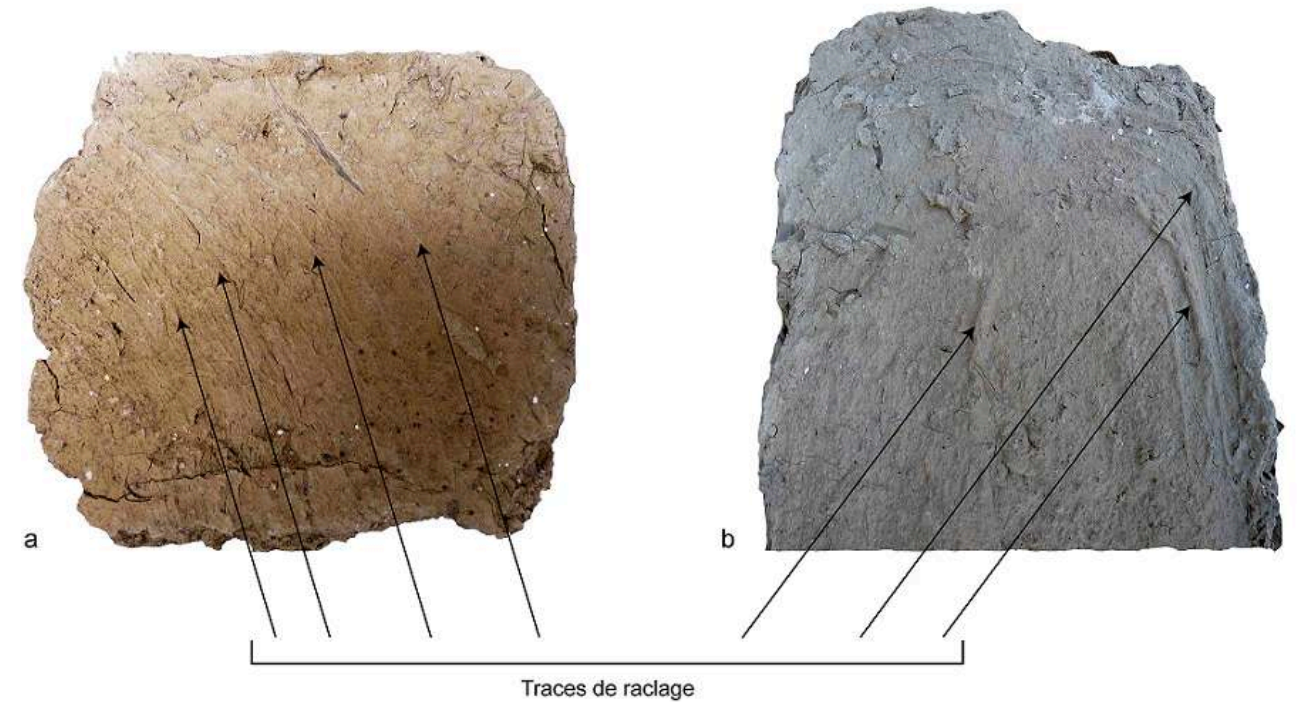

Description : Les traces de raclage sur la face supérieure des briques de Mentesh Tepe datées du Chalcolithique (5e millénaire), sont caractéristiques de l'étape de l'enlèvement de l'excédent de terre par l'artisan avant le retrait du moule.

Mission Mentesh Tepe

11 Deuxièmement, le retrait du moule par le haut laisse généralement un bourrelet de terre sur la partie supérieure de la brique ou des traces d'écrêtage sur les tranches sommitales (Fig. 3). Le même type de stigmate peut être observé sur la partie inférieure des briques façonnées selon la technique du "moule enfoncé » lors du débitage des briques par enfoncement $d u$ moule dans la masse de terre préalablement préparée (Aurenche $1981: 65)^{3}$. D'autres observations peuvent également apporter des précisions sur certaines étapes de la confection ${ }^{4}$, comme la présence de négatifs de végétaux sur l'une des faces de la brique, signe que la terre à bâtir a été étalée sur un lit végétal avant la fabrication des briques (Daune-Le Brun 2003 :156). 
Fig.3 : Mentesh Tepe, Azerbaïdjan (Chalcolithique, $5^{\mathrm{e}}$ millénaire). Stigmates laissés lors du retrait du moule et affaissement des parois

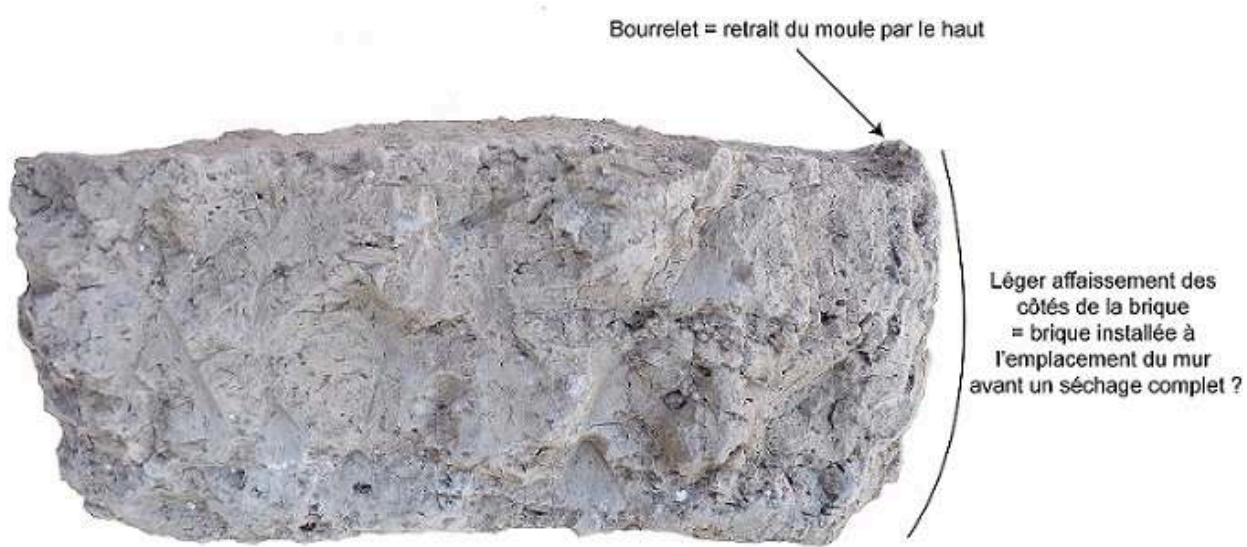

Le bourrelet de terre présent sur la face supérieure de la brique témoigne de l'étape du retrait du moule par le haut. Quant à l'affaissement des parois de la brique, elles montrent que le mur a dû être monté avant le séchage complet des briques crues.

Mission Mentesh Tepe

Parmi les critères secondaires, retenons la régularité des formats ${ }^{5}$, la présence d'angles et d'arêtes nets et des côtés de la brique rectilignes. Si la récurrence de ces observations sur une même brique ne laisse que peu de doute quant à l'interprétation de l'utilisation du moule, il faudra privilégier celle du modelage en l'absence de ces indices (Tunca 1984 :122). Toutefois, au regard de la maitrise des constructeurs anciens confectionnant des briques modelées (Aurenche $1981: 64$ ), retenir les seuls critères secondaires n'est pas suffisant pour définir avec certitude l'usage du moule.

Ces observations de terrain peuvent être complétées par des analyses micromorphologiques. L'examen au microscope apporte des précisions sur la composition, le degré d'humidité et l'intensité du malaxage lors de la préparation de la terre à bâtir (Cammas 2003). L'analyse des lames minces renseigne également sur la technique de façonnage utilisée. Par exemple, l'observation de longues fissures rectilignes et de "vides aplatis» (Cammas 2015 :63) est caractéristique d'une brique moulée (Tab.1). 
Tableau1 : récapitulatif de la chaîne opératoire des différentes techniques de façonnage de la brique (en rouge, les éléments observables sur le terrain et en violet, les éléments observables par une analyse micromorphologique).

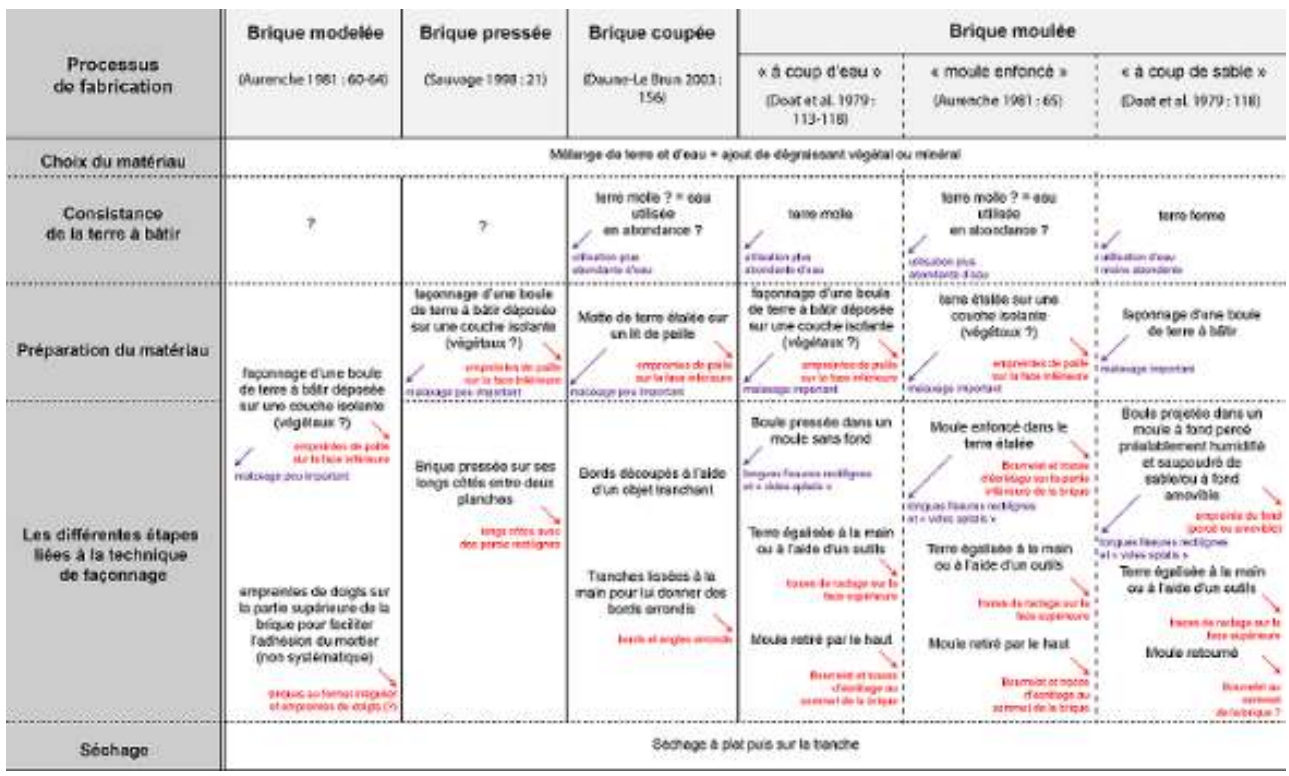

À chacune des étapes de la fabrication d'une brique correspondent des stigmates particuliers. Ces stigmates peuvent être observés à l'œil nu, directement sur le terrain lors de la fouille, où, pour certains, nécessitent une analyse microscopique du matériau de construction en laboratoire par une étude micromorphologique.

Emmanuel Baudouin

\section{Finalement, modelage ou moulage?}

14 Les dernières découvertes font état de l'utilisation de la brique modelée dans la plaine de Mil, à Kamiltepe (Helwing et al. 2017 :17). Ces briques, employées pour la construction d'une terrasse entièrement en brique de $24 \mathrm{~m}$ de diamètre et préservée sur 2,6m de hauteur, ont été identifiées comme telles par leur format irrégulier. Ce même type de briques est aussi attesté dans la moyenne vallée de la Kura, à Mentesh Tepe, mais seulement pour la construction d'une petite installation circulaire constituée de briques posées de chant et non pour l'édification des murs (Baudouin et al. $2017: 44$ ). Ces briques ont des angles et des bords très arrondis et des dimensions variables.

En revanche, les données inédites récoltées dans la moyenne vallée de la Kura à Aruchlo et à Mentesh Tepe font état de l'utilisation de briques plano-convexes moulées dès le début du $6^{\mathrm{e}}$ millénaire. À Mentesh Tepe, les observations de terrain ont montré que certaines avaient des rebords parfaitement droits et des bourrelets à leur sommet (Fig.4 a-b) pour des dimensions constantes de 43-45x15-16x9-10cm (Baudouin2019: 112, tab.3). La présence d'un bourrelet sur la partie inférieure de la brique pourrait expliquer l'usage de la technique du "moule enfoncé ", même s'il est difficile de trancher en l'absence de données quantitatives suffisantes ${ }^{6}$. 
Fig.4 a-b : Mentesh Tepe, Azerbaïdjan (Néolithique, début du 6e millénaire). Bourrelets laissés lors du retrait du moule sur une brique crue plano-convexe moulée

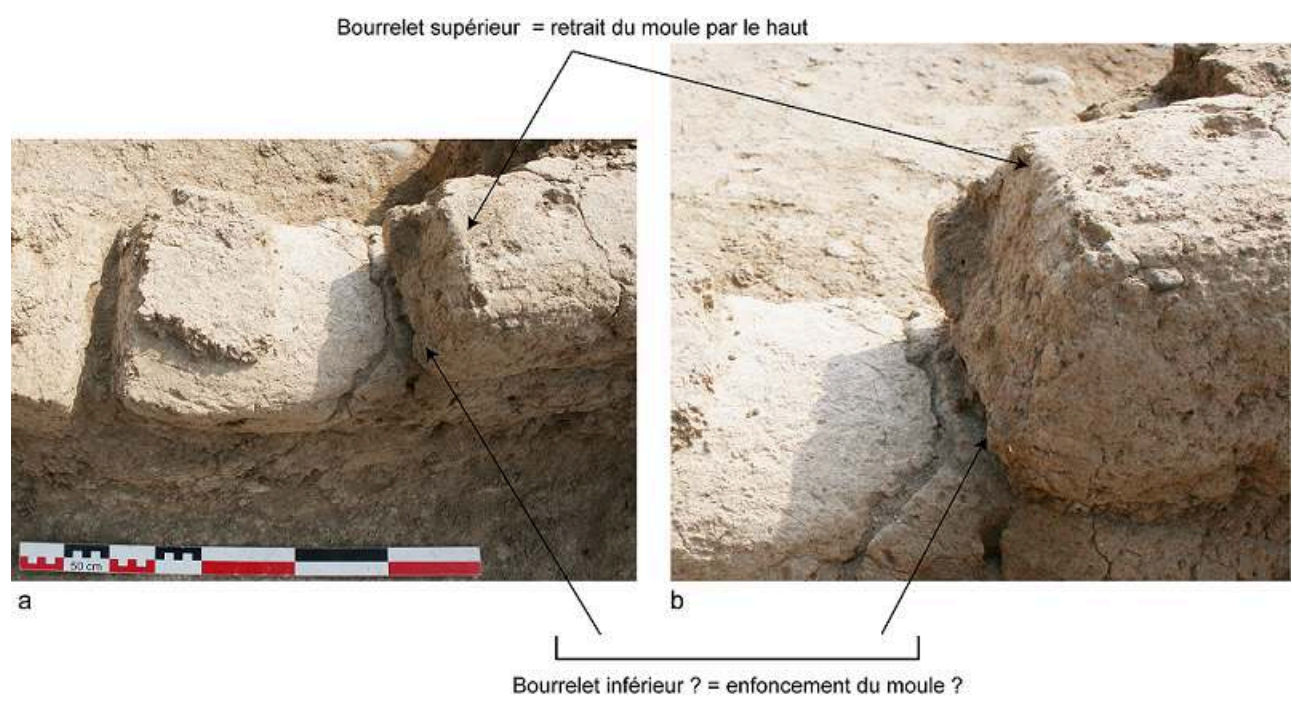

Les deux bourrelets de terre, sur la face supérieure et la face inférieure de la brique sont les stigmates caractéristiques d'une technique particulière de la brique moulée, celle dite "à moulée enfoncée ». L'enfoncement du moule sur la terre préalablement étalée crée, à la base de la brique, un premier bourrelet de terre. Ensuite, le retrait du moule par le haut crée un second bourrelet de terre sur la partie supérieure de la brique.

Mission Mentesh Tepe

16 Par ailleurs, de légères stries longitudinales (Fig.5 a-b) sur la face bombée de plusieurs briques laissent à penser qu'une égalisation de la surface a été faite à l'aide d'un outil ou du tranchant de la main (Baudouin 2019) à moins que ces traces n'aient été destinées à faciliter l'adhésion du mortier lors de l'érection du mur (Aurenche $1981: 62$; Sauvage $1998: 41-42$ ) ou les deux à la fois.

Fig.5 : Mentesh Tepe, Azerbaïdjan (Néolithique, début du $6^{\mathrm{e}}$ millénaire). Traces supposées de raclage sur la face supérieure d'une brique crue moulée plano-convexe
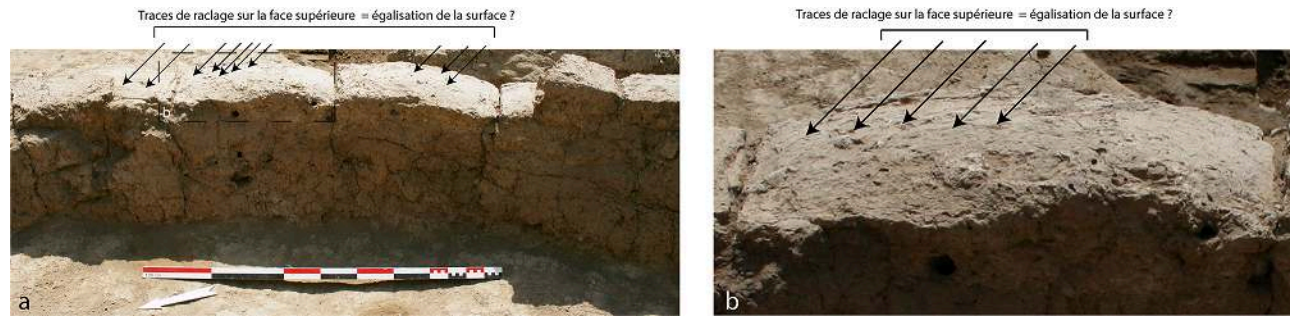

Les traces supposées de raclage sur la face supérieure de cette brique plano-convexe sont attribuables soit à un raclage de la surface pour enlever l'excédent de terre avant l'enlèvement du moule, soit à des traces laissées volontairement sur la surface pour faciliter, lors du montage du mur, l'adhésion du mortier.

Mission Mentesh Tepe

17 À Aruchlo, des stigmates similaires ont pu être observés sur des briques cuites accidentellement retrouvées dans un niveau d'effondrement incendié associé au début de l'occupation du site (Ioseliani 2017 :282). Comme à Mentesh Tepe, la présence probable d'un bourrelet de terre à la base de certaines briques permettrait de privilégier l'hypothèse de l'utilisation de la technique du «moule enfoncé » (Fig. 6 a) ${ }^{7}$. 
Fig. 6 : a) Aruchlo, Géorgie (Néolithique, début du $6^{\mathrm{e}}$ millénaire). Stigmates caractéristiques d'une brique moulée avec bourrelet supérieur, bourrelet inférieur et parois rectilignes (d'après Ioseliani $2017: 206$, fig. 12) ; b) Section d'une brique plano-convexe (d'après Delougaz 1933 : 5, fig. 4)

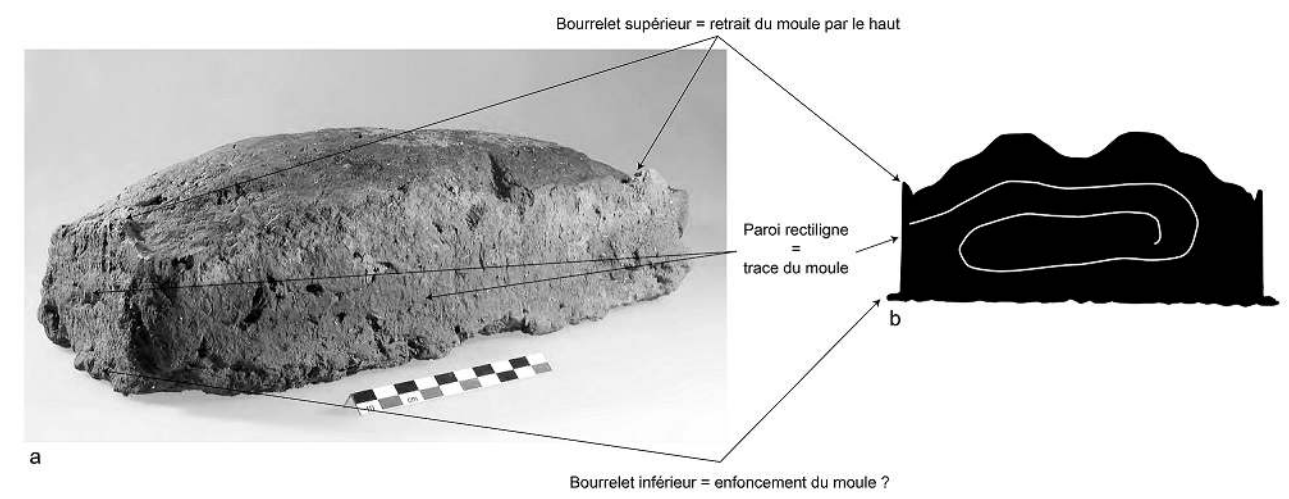

Des parois rectilignes, un bourrelet sur la partie supérieure de la brique, un second sur la partie inférieure de la brique, sont autant d'indices de la technique de la brique moulée dite « du moule enfoncée » qui consiste à enfoncer le moule sur la terre préalablement étalée sur le sol puis à le retirer ensuite par le haut.

a) d'après Ioseliani 2017 : 206, fig. 12 ; b) d'après Delougaz 1933 : 5, fig. 4.

\section{La carte d'identité de la brique moulée}

L'emploi du moule est une innovation majeure désormais bien documentée au ProcheOrient (Sauvage $2009: 194$, fig.4). La technique semble apparaître au PPNB récent (6600-6000 av. J.-C.) en Anatolie, avant de se diffuser dans la moyenne vallée de l'Euphrate puis en Mésopotamie centrale durant le PPNB final et le Samarra (6000-5700 av. J.-C.). Quant au Caucase, l'enjeu est de déterminer s'il s'agit d'une innovation autonome ou, au contraire, d'une importation venue de Mésopotamie. Il est donc nécessaire de considérer deux paramètres principaux: la géographie et la chronostratigraphie.

Dans l'état actuel des données, l'absence de briques moulées dans la vallée de l'Araxe constitue un problème dans l'hypothèse d'un transfert technique venu de Mésopotamie. En effet, cette région est un point de passage obligatoire entre la plaine mésopotamienne et la vallée de la Kura. Expliquer le rejet de son adoption dans la vallée de l'Araxe s'avère difficile tant cette innovation représente un progrès technique majeur, à moins de considérer que les populations n'étaient pas prêtes au changement. Dans ce cas, cette «inertie technique " (Leroi-Gourhan 1945, 323) serait le reflet du refus d'assimilation d'une nouvelle technique. Une autre hypothèse serait de considérer que cette particularité représente une simplification des techniques (Pétrequin, Cassen 2018). Dans ce cas, cette adaptation serait le signe que le « culturel » aurait influé directement sur le traitement de la matière (Lemonnier 1991 :19).

Par ailleurs, la forme plano-convexe des briques de la vallée de la Kura ne trouve pas de parallèle tant dans la vallée de l'Araxe qu'en Mésopotamie ${ }^{8}$. Or, la morphologie semble avoir une importante valeur technico-culturelle au regard de son usage bien circonscrit. Cette absence de comparaison fragilise davantage l'interprétation d'une diffusion technique venue de Mésopotamie. 
21 La présence de briques moulées dès la phase formative du Néolithique atteste de la précocité de cette technique dans la vallée de la Kura. Les données stratigraphiques à Mentesh Tepe et à Aruchlo tendent à montrer que la brique moulée est utilisée dès les premières phases d'occupation sur ces deux sites. Selon les datations par radiocarbone, ces niveaux sont respectivement datés aux alentours de 5990-5790 av. J.-C. (Lyonnet et al. 2017 :128, tab.1) et de 5880-5730 av. J.-C. (Hansen et al. 2012 :85, tab.19).

Par ailleurs, l'occupation primitive de plusieurs sites de la vallée de la Kura est caractérisée par l'adoption d'une architecture semi-enterrée (Fig. 7) comme à Mentesh Tepe, Aruchlo, Shomu Tepe ou encore à Haci Elamxanli Tepe (Baudouin 2019 :134-137).

Fig.7 : Mentesh Tepe, Azerbaïdjan (Néolithique, début du 6 $6^{\mathrm{e}}$ millénaire). Bâtiment semi-enterré 1031 (Baudouin et al. $2017: 45$, fig. 3)

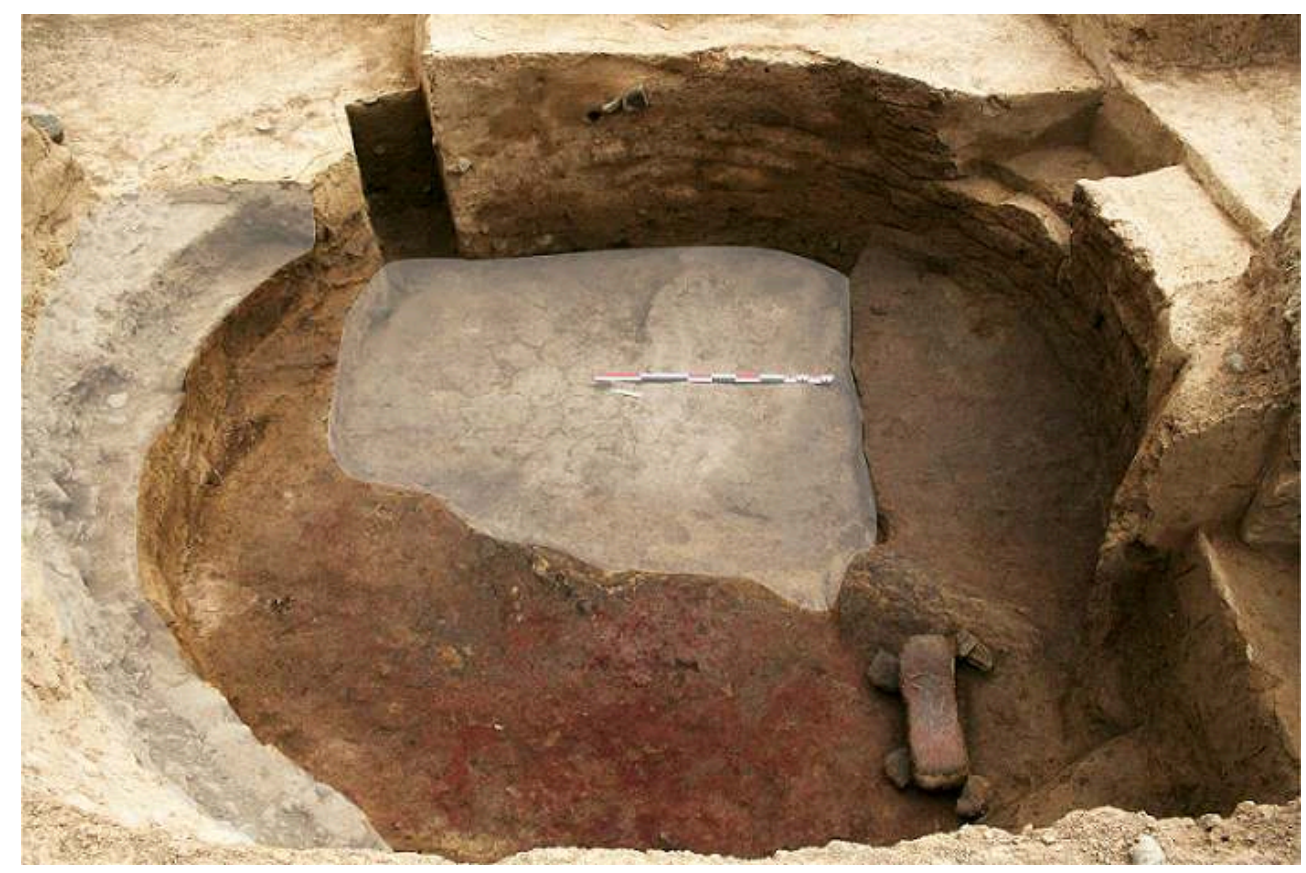

L'architecture semi-enterrée est présente dans le Caucase au Néolithique dans les niveaux les plus anciens des sites de la moyenne vallée de la Kura, au début du 6e millénaire. Ce type d'architecture semble être lié au début de la sédentarisation dans la région et pourrait correspondre à l'installation de populations encore peu familières avec les techniques architecturales.

Mission Mentesh Tepe

Or, le développement de ce type architectural est un phénomène bien documenté par l'archéologie et l'ethnographie. Son utilisation est couramment associée à l'adoption récente d'un mode de vie sédentaire par des populations anciennement nomades (Aurenche 1981 :98; Cauvin 1978 :23) et coïncide généralement avec un développement local des techniques architecturales. Si, à Aruchlo, la contemporanéité de l'architecture semi-enterrée et l'utilisation des briques plano-convexes n'a pu être prouvée par la stratigraphie', à Mentesh Tepe, ces deux techniques sont concomitantes. S'il n'est pas possible d'établir un lien de cause à effet entre le développement de l'architecture semi-enterrée et celui de la brique moulée, l'originalité morphologique et technique des briques plano-convexes renforce l'hypothèse d'une genèse autonome des techniques architecturales dans la vallée de la Kura. Dans une autre mesure, les attestations de briques modelées dans cette région permettraient de documenter une invention autonome. Néanmoins, dans l'état actuel des connaissances, la succession 
clayonnage/bauge/brique bien attestée en Mésopotamie ne peut être prouvée dans le Caucase. Il conviendrait alors de considérer que le développement technique des communautés de la vallée de la Kura est imputable au milieu intérieur (Leroi-Gourhan 1945 :322), indépendamment de toute influence culturelle extérieure en ce domaine.

\section{Perspectives}

Ce bref tour d'horizon permet d'ores et déjà de confirmer l'utilisation de la brique crue dès la fin du $7^{e}$ millénaire dans le Caucase. Si les informations quant au mode de façonnage font encore défaut dans la plupart des cas, les découvertes récentes à Aruchlo et à Mentesh Tepe apportent un éclairage nouveau pour comprendre l'évolution des techniques dans la région.

La brique crue moulée apparaît dès la phase formative du Néolithique. Dans l'état actuel des recherches, son utilisation semble limitée à la vallée de la Kura et pourrait coïncider avec une genèse locale de la technique. Cette interprétation est confortée par la présence d'une architecture semi-enterrée et de briques modelées dans les phases les plus anciennes sur plusieurs sites de la région, indice probable d'une sédentarisation récente et autonome de populations auparavant nomades.

Ce travail représente une première étape vers une meilleure compréhension de l'évolution des techniques architecturales dans le Caucase. Ces résultats devront être confirmés par de nouvelles recherches sur le terrain. Il serait notamment intéressant de déterminer le mode de façonnage des briques dans la vallée de l'Araxe. Enfin, l'utilisation de la micromorphologie s'avère désormais indispensable lors de fouilles archéologiques. Ce travail devrait passer par la réalisation d'un échantillonnage et d'un examen systématique des matériaux de construction sur l'ensemble des sites fouillés.

\section{BIBLIOGRAPHIE}

ABIBULLAEV Osman A., Arkheeologicheskie rakopki v Kyultepe [Archaeological excavations at Kültepe], Baku, 1959

AMIRKHANOV Khizri A., Chokhskoe poselenie - Chelovek i ego kul'tura $v$ nezolite i neolite gornogo DagestanaI [The Chokh settlement. Men and culture in the Mesolithic and Neolithic of mountainous Dagestan], Moscou, 1987

AURENCHE Olivier, La maison orientale : l'architecture du Proche-Orient ancien des origines au milieu du quatrième millénaire, Paris, P. Geuthner, 1981

AURENCHE Olivier, «L'origine de la brique dans le Proche-Orient ancien », in FRANGIPANE Marcella, HAUPTMANN Harald, LIVERANI Mario, MATTHIAE Paolo, MELLINK Machteld (eds), Between the Rivers and Over the Mountains: Archaeological Anatolica et Mesopotamica. Alba Palmieri dedicata, Rome, Dipartimiento di Scienze Storiche Archeologiche e Anthropologiche dell'Antichità, Università di Roma La Sapienza, 1993, p.71-86 
AURENCHE Olivier, « Propositions de terminologie pour les modalités de mise en œuvre de la terre comme matériau de construction » in CHAZELLES C.-A. et KLEIN A. (eds), Échanges transdisciplinaires sur les constructions en terre. Actes de la table-ronde de Montpellier 17-18 novembre 2001. 1. Terre modelée, découpée ou coffrée : matériaux et modes de mise en cuvre, Montpellier, Éd. de l’Espérou, 2003, p.279-282

BAUDOUIN Emmanuel, « Remarques préliminaires sur les techniques architecturales du Néolithique et du Chalcolithique à Mentesh Tepe (Azerbaijan) » in HELWING Barbara, ALIYEVl Tevekkül, LYONNET Bertille, GULIYEV Farhad, HANSEN Svend and MIRTSHULAVA Guram (eds.), The Kura Projects: New Research on the Late Prehistory of the Southern Caucasus. Archäologie in Iran und Turan, Berlin, Deutsches Archäologisches Institut, Eurasien-Abteilung, 2017, p.153-162

BAUDOUIN Emmanuel, « Rethinking Architectural Techniques of Southern Caucasus in the 6th Millennium BC: A Re-Examination of Former Data and New Insights ", Paléorient 45/1, 2019, p. 101-136

BAUDOUIN Emmanuel, L'architecture en Syro-Mésopotamie et dans le Caucase de la fin du $7^{e} \grave{a}$ la fin du $5^{e}$ millénaire, Turnhout, Brepols Publishers (sous presse)

BAUDOUIN Emmanuel, LYONNET Bertille, HAMON Caroline, « Architectural Techniques and Cultural Relationships between the Caucasus and Mesopotamia at the Beginning of the Sixth Millennium B.C » in DOUCHÉ Carolyne et PICHON Fionia (eds), Routes de l'Orient, Actes I. From the Caucasus to the Arabian Peninsula: Domestic Spaces in the Neolithic, 2018, p.49-84

BAUDOUIN Emmanuel, MURA Mathilde, MANEL Camille, « Mentesh Tepe (Azerbaïdjan) : résultats préliminaires de la campagne de fouille 2015 », Routes de l'Orient, 3, 2017, p.41-52

CAMMAS Cécilia, « L'architecture en terre crue à l'âge du Fer et à l'époque romaine : apports de la discrimination micromorphologique des modes de mise en œuvre ", in CHAZELLES Claire-Anne de, KLEIN Alain (éds), Échanges transdisciplinaires sur les constructions en terre. Actes de la table-ronde de Montpellier 17-18 novembre 2001. 1. Terre modelée, découpée ou coffrée : matériaux et modes de mise en œuvre, Montpellier, Éd. de l'Espérou, 2003, p.33-53

CAMMAS Cécilia, « La construction en terre crue de l'âge du Fer à nos jours. L'apport de la micromorphologie à la compréhension des techniques ", Archéopages. Archéologie et société, 42, 2015, p.58-67

CAUVIN Jacques, Les Premiers villages de Syrie-Palestine $d u$ IXème au VII ème millénaire avant J.C., Lyon, Maison de l'Orient, 1978

CHATAIGNER Christine, La Transcaucasie au Néolithique et au Chalcolithique, Oxford, Tempus Reparatum, 1995

DARLES C., ROUX J.-C., BRETON J.-F., " L'architecture en brique crue au Yémen de l'Antiquité à nos jours » in CHAZELLES Claire-Anne de, KLEIN Alain et POUSTHOMIS-DALLE Nelly (éds), Les cultures constructives de la brique crue. Échanges transdisciplinaires sur les constructions en terre crue-3, Montpellier, Éd. de l'Espérou, 2011, p.13-29

DAUNE-LE BRUN Odile, « La terre à bâtir, son emploi dans les constructions du Néolithique précéramique récent de Chypre ", in CHAZELLES Claire-Anne de, KLEIN Alain (éds), Échanges transdisciplinaires sur les constructions en terre. Actes de la table-ronde de Montpellier 17-18 novembre 2001. 1. Terre modelée, découpée ou coffrée : matériaux et modes de mise en cuvre, Montpellier, Éd. de l'Espérou, 2003, p.151-160

DELOUGAZ Pinhas, Plano-convex Bricks and Method of their Employment, Chicago, The Oriental Institute of the University of Chicago, 1933 
GUEST-PAPAMANOLI Anna, «L'emploi de la brique crue dans le domaine égéen à l'époque néolithique et à l'Âge du Bronze », Bulletin de correspondance hellénique, 102/1, 1978, p.3-24

HANSEN Svend, MIRTSKHULAVA Guram, « The datings », in LYONNET Bertille, GULIYEV Farhad, HELWING Barbara, ALIYEV Tevekkül, HANSEN Svend and MIRTSKHULAVA Guram (eds.), Ancient Kura 2010-2011: The first two seasons of joint field work in the southern Caucasus. Archäologische Mitteilungen aus Iran und Turan, 44, 2012, p.85-86

HELWING Barbara, ALIYEV Tevekkül, « Excavations in the Mil Plain sites, 2012-2014 ", in HELWING Barbara, ALIYEV Tevekkül, LYONNET Bertille, GULIYEV Farhad, HANSEN Svend and MIRTSHULAVA Guram (eds.), The Kura Projects: New Research on the Late Prehistory of the Southern Caucasus. Archäologie in Iran und Turan, Berlin, Deutsches Archäologisches Institut, EurasienAbteilung, 2017, p.11-42

IOSELIANI Vladimir, « Architecture Debris from the Burnt Layers », in HELWING Barbara, ALIYEV Tevekkül, LYONNET Bertille, GULIYEV Farhad, HANSEN Svend and MIRTSHULAVA Guram (eds.), The Kura Projects: New Research on the Late Prehistory of the Southern Caucasus. Archäologie in Iran und Turan, Berlin, Deutsches Archäologisches Institut, Eurasien-Abteilung, 2017, p.281-286

KIGURADZE Tamaz, Neolithische Siedlungen von Kvemo-Kartili, Georgien, München, Beck, 1986

KUSHNAREVA Kariné Ch., The Southern Caucasus in Prehistory: Stages of Cultural and Socioeconomic Development from the Eighth to the Second Millennium BC, Philadelphia: University Museum, University of Pennsylvania, 1997

LEMONNIER Pierre, « De la culture matérielle à la culture ? Ethnologie des techniques et Préhistoire ", Collectif, 25 ans d'études technologiques en Préhistoire, bilan et perspectives, Juan-lesPins, APDCA, 1991, p.15-20

LEROI-GOURHAN André, Évolution et techniques [2]. Milieu et techniques, Paris, Albin Michel, 1945

LYONNET Bertille, GULIYEV Guliyev, in collaboration with Emmanuel BAUDOUIN, Laurence BOUQUET, Gaëlle BRULEY-CHABOT, Annaïk SAMZUN, Michel FONTUGNE, Émeline DEGORRE, Xavier HUSSON and Pascal RAYMOND, « Mentesh Tepe (Azerbaïdjan) a preliminary report on the 2012-2014 excavations », dans HELWING Barbara, ALIYEVl Tevekkül, LYONNET Bertille, GULIYEV Farhad, HANSEN Svend and MIRTSHULAVA Guram (eds.), The Kura Projects: New Research on the Late Prehistory of the Southern Caucasus. Archäologie in Iran und Turan, Berlin, Deutsches Archäologisches Institut, Eurasien-Abteilung, 2017, p.125-140

NARIMANOV I.N., Kul'tura Drevneyshego Zemledel'chesko-Skotovocheskogo Naseleniya Azerbadzhana, Epokho Eneolita VI-IV tys. do n.e. [The culture of the earliest farmers and herders in Azerbaijan, Chalcolithic period 6th-4th millenia BC], Baku, Elm, 1987

PETREQUIN Pierre, CASSEN Serge, « Chapitre 7. Des innovations techniques pendant le Néolithique et leurs représentations sociales », GUILAINE Jean, GARCIA Dominique, La Protohistoire de la France, Paris, Hermann Histoire et Archéologie, 2018, p.127-140

SAUVAGE Martin, La brique et sa mise en œuvre en Mésopotamie : des origines à l'époque achéménide, Paris, ERC, 1998

SAUVAGE Martin, « Les briques de grande taille à empreintes de doigts : le Choga Mami Transitional et la culture de Oueili », KEPINSKI Christine et BRENIQUET Catherine (eds), Études mésopotamiennes : Recueil de textes offert à Jean-Louis Huot, Paris, ERC., 2001, p.417-447

SAUVAGE Martin, "Les débuts de l'architecture de terre au Proche-Orient », ACHENZA Maddalena, CORREIA Mariana, GUILLAUD Hubert (eds), L'architettura in terra cruda del 
Mediterraneo. Prima Conferenza mediterranea sull'architettura in terra cruda, Cagliari, Edicom Edizioni, 2009, p.189-198

SAUVAGE Martin, «L'architecture de brique crue en Mésopotamie », CHAZELLES Claire-Anne de, KLEIN Alain et POUSTHOMIS Nelly (éds), Les cultures constructives de la brique crue. Échanges transdisciplinaires sur les constructions en terre crue-3, Montpellier, Éd. de l'Espérou, 2011, p.89-100

TUNCA Önhan, L'architecture religieuse protodynastique en Mésopotamie, Louvain, Peeters, 1984

VAVILOV Nikolai I., « Problemy proiskhozhdeniya mirovogo zemledeliya v svete sovremennykh issledovaniy [Issues concerning the origin of world agriculture in the light of contemporary investigations] », Doklady sovetskikh delegatov na II Mezhdunarodnom kongresse po istorii nauki i tekhniki, London, 1931. Izbrannye strudy, Moscou, Leningrad, 1932, p.143-152

WULFF Hans E., The Traditional Crafts of Persia: their Development, Technology, and Influence on Eastern and Western Civilizations, Cambridge, The M.I.T. press, 1966

\section{NOTES}

1. À la différence de la brique qui est un élément préfabriqué, la bauge est modelée directement à l'emplacement du mur.

2. Ces traces peuvent être laissées par un outil ou, plus simplement, comme cela est souvent le cas, par le tranchant de la main.

3. Ce type de stigmate peut aussi être retrouvé sur certaines briques confectionnées selon la technique « à coup d'eau » où, lors du pressage de la terre dans le moule afin de bien combler les angles, le contenu peut légèrement déborder par le bas.

4. Comme indiqué dans le tableau 1 , ces stigmates sont indépendants de la technique du façonnage car le but recherché, d'éviter que le matériau adhère au sol, est commun à toutes les techniques.

5. Olivier Aurenche faisait remarquer que le savoir-faire des briquetiers produisant des briques modelées est tel que les différences dans la dimension sont infimes (Aurenche 1981 : 64).

6. Dans la mesure où ces stigmates n'ont été dûment observés que sur quelques briques et que nous avons vu précédemment qu'ils pouvaient être aussi observés pour la technique «à coup d'eau », cette interprétation doit être nuancée.

7. Selon Anna Guest-Papamanoli (1978), le critère principal qui permet d'identifier la technique à "moule enfoncé ", outre le moulage, est la disposition d'un boudin sur le sol qui correspond au fait d'enrouler la pâte. C'est d'ailleurs ce type de stigmate qui a semble-t-il été observé sur certaines briques plano-convexes moulées du Dynastique Archaïque (2900-2340 av. J.-C.) en Mésopotamie (Fig. 6b). Il serait intéressant de réaliser des expérimentations archéologiques afin de confirmer ou non cette hypothèse.

8. La seule attestation présumée de brique plano-convexe en Mésopotamie vient du site de Tell Arpachiyah durant la période de Halaf (6400-5400 av. J.-C.) mais aucune photographie ou dessin ne permet de justifier son usage.

9. Les bâtiments semi-enterrés d'Aruchlo ont été fouillés dans les années 1960. La reprise de la stratigraphie des fouilles anciennes est actuellement en cours d'étude par Vladimir Ioseliani. 


\section{RÉSUMÉS}

Si l'origine des premières communautés sédentaires du Caucase à la fin du 7e millénaire est toujours débattue, une reprise récente des données archéologiques sur les techniques architecturales tend à montrer que les relations culturelles avec la Mésopotamie sont complexes. L'évolution de la technique de la brique crue illustre parfaitement cette ambivalence. Les découvertes archéologiques effectuées à Aruchlo et à Mentesh Tepe confirment l'emploi de la technique du moulage dès la phase formative du Néolithique. L'origine de cette innovation technique semble être le fruit d'une invention autonome et non d'un transfert de savoir-faire venu de Mésopotamie. Cet article est aussi l'occasion de faire un bilan des moyens à disposition des archéologues pour reconnaitre les différentes techniques de façonnage de la brique sur le terrain.

If the birth of the first sedentary communities in the Southern Caucasus at the end of the 7th millennium $\mathrm{BC}$ is discussed, a resumption of archaeological data on architectural techniques demonstrates that cultural relationships with Mesopotamia are complex. The evolution of mud brick technique illustrates this inconsistency. Recent archaeological discoveries at Aruchlo and Mentesh Tepe attest the use of moulding since the beginning of Neolithic. The origin of this technical innovation is probably the result of an autonomous invention and not of a know-how transfer from Mesopotamia. This article is also an opportunity to review the resources available to archaeologists to identify the different manufacturing techniques on the field.

\section{INDEX}

Mots-clés : histoire des techniques, brique, archéologie, moulage, archéologie du bâti, construction, modelage, matériau

Keywords : history of technology, materials, archeology, mud brick, building, moulding, modelling

Thèmes : Un objet une technique

\section{AUTEUR}

\section{EMMANUEL BAUDOUIN}

Emmanuel Baudouin est chercheur associé à l'UMR 7041 ArScAn. Ses recherches portent sur les processus d'innovations, d'inventions et de diffusions techniques dans le domaine de l'architecture entre les communautés de Mésopotamie et celles du Caucase durant le Néolithique et le Chalcolithique. C'est au travers d'une analyse détaillée de l'évolution des techniques en architecture qu'il essaye de comprendre les mécanismes liés aux transferts techniques et aux échanges de savoir-faire entre les communautés. Suite à un doctorat soutenu en 2018 à Sorbonne Université, ses activités de recherche se sont poursuivies à l'Université de Tokyo en 2018-2019 dans le cadre d'une bourse postdoctorale, grâce au financement de la Japan Society of the Promotion of Science (JSPS). Un article de synthèse intitulé « Rethinking architectural techniques of the Southern Caucasus in the 6th millennium BC : A re-examination of former data and new insights ", publié dans la revue Paléorient ( $\left.\mathrm{n}^{\circ} 45.1\right)$, permettra au lecteur d'approfondir la lecture sur le sujet présenté ici. 